\title{
The frail elderly and integral health management centered on the individual and the family
}

The rapid aging of the Brazilian population, combined with an increase in longevity, has had serious consequences for the structure of health care networks, with an increased burden of chronic diseases and especially of functional disabilities. Unfortunately, the care offered to frail elderly people with multiple chronic health conditions, poly-disabilities or complex needs is fragmented, inefficient, ineffective and discontinuous, which can further harm their health. The hospital-based health system of the $19^{\text {th }}$ and $20^{\text {th }}$ centuries that is designed to deal with acute and especially infectious diseases is inadequate for meeting the needs of chronic patients for long-term, continuous treatment. The response of the health system to the new demands means the use of a set of management technologies that are capable of ensuring optimal standards of health care in a resolutive, efficient, scientifically structured manner, which is safe for patients and health professionals, timely, equitable, humanized and sustainable, is essential. The threefold goal of a better care experience, coupled with improved populational health and reduced costs developed by the Institute of Healthcare Improvement (Triple Aim), is the best strategy for reorganizing and optimizing health system performance.

Providing the best care experience means understanding the particularities of health in the elderly. The use of parameters based on risk factors, diseases and/or age is inappropriate and is associated with a high risk of iatrogenic illness. Vitality is extremely heterogeneous among the elderly and chronological age is a precarious metric for the assessment of the homeostatic reserve of the individual. Likewise, aging without suffering any chronic illnesses is the exception rather than the rule. Knowing only the age of individuals and their diseases, therefore, does not improve the chances of a better understanding of the health situation. The best marker of vitality in the elderly is functional capacity, as measured by activities of daily living (ADL). It represents autonomy (individual decision making capacity) and independence (the ability to execute the activity), allowing individuals to take care of themselves and their lives. Thus, health in the elderly can be defined as the individual's ability to satisfy their biopsychosocial needs, regardless of age or the presence of diseases. The decline in vitality is known as frailty, clearly associated with the development of disabilities, functional dependence, hospitalization and death. Therefore, the presence of functional decline in activities of daily living should never be attributed to aging itself and should always trigger an extensive investigation of the elderly person. This should consider the performance of functional systems (cognition, mood/behavior, mobility and communication) and physiological systems (sleep, nutrition, oral health, skin and annexes, cardiovascular, respiratory, digestive, genitourinary, musculoskeletal, nervous and endocrine-metabolic systems), together with an evaluation of the medications being used, previous history and contextual factors, compiled from a socio-family assessment, the evaluation of the caregiver and an environmental assessment. This Multidimensional Evaluation of the Elderly Person is fundamental for the construction of a Personalized Care Plan (PCP), or the set of biopsychosocial and functional diagnoses of 
the individual, associated with promotional, preventive, curative, palliative and/or rehabilitative interventions capable of maintaining or recovering their health. The PCP is, therefore, the guiding strategy for the health team to meet specific patient health needs in the short, medium and long term, and must also be strongly anchored in the principles of Patient-Centered Medical Home. This should be the logic that directs the team in the search for suitable resources and required treatments, serving as the guiding axis of the entire care process. The proposed interventions should also be applied based on the vitality of the elderly person, which may be robust, at risk of frailty or frail.

Frail elderly persons are the largest consumers of procedures in any health system, with these processes for the most part being associated with iatrogenic illness and therapeutic futility. Health promotion strategies and primary and secondary prevention measures should be applied with great caution as the impact of such measures requires behavioral and lifestyle changes, as well as investments in diagnostic and therapeutic interventions that take far too long to obtain positive results. This type of frail elderly person does not have a sufficiently long life expectancy to benefit from such interventions, which also present risks and can have negative repercussions for their health. Tertiary prevention, which consists of the recognition of disabilities and interdisciplinary interventions aimed at rehabilitation, demands time, qualified professional care and patient and family adherence, which are all associated with high costs. Recently the concept of quaternary prevention has been introduced, which consists of detecting individuals at risk of excessive treatment to protect them from further inappropriate medical interventions and suggest ethically acceptable alternatives. Quaternary prevention is aimed at protecting people who use the health system from an excess of disease-tracking interventions, the medicalization of risk factors, the request for too many exams, overdiagnosis, and the use of futile or potentially inappropriate and defensive medicine. Its main purpose is not to harm the patient ("primum non nocere"). These health micromanagement concepts are fundamental for the maintenance and/or recovery of the independence and autonomy of the elderly, and also for the sustainability of health care systems, whether public or private (health macromanagement). From this perspective, the main interventions capable of improving the health of frail elderly persons are, in order of importance: a) suspension of inadequate diagnostic and/or therapeutic interventions, with emphasis on the safe deprescription of inappropriate drugs; b) the definition of individualized therapeutic goals shared with the elderly and their family, respecting the clinical-functional stratum of the patient; $(\mathrm{C})$ appropriate treatment of underdiagnosed and, consequently, under-treated conditions, commonly attributed to "age" or "senility"; D) rehabilitation, according to the patient's rehabilitation prognosis; E) secondary prevention when life expectancy is sufficiently long for the expected benefit to occur and there are no contraindications in the medication, dietary or lifestyle changes and f) primary prevention, only when the elderly individual is robust and the cost-benefit-risk ratio of the treatment is highly satisfactory, due to the scarcity of intervention studies among very old elderly persons and especially of the frail elderly.

All these premises are included in the proposed Gestão Integral da Saúde Centrada no Idoso e na Família (Integrated Health Management for the Elderly and the Family) (GISCI) model, which is capable of reconciling health micromanagement technologies and the more modern premises of clinical macromanagement, implemented in an integral and integrated form. Both technologies must be applied simultaneously to ensure the effectiveness and sustainability of the model. GISCI is being progressively installed in the SUS (the Brazilian Unified Health System), through the experiences of the Centro de Referência do Idoso (the Elderly Reference Center) of the Hospital das Clínicas of the Federal University of Minas Gerais (UFMG) and in Supplementary Health, with the Usifamília program of the Fundação São Francisco Xavier, both of which have demonstrated highly promising results. 